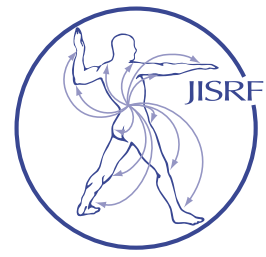

\title{
Perioperative Complications Following One-Stage Bilateral and Unilateral Total Hip Arthroplasty via Direct Anterior Approach
}

\section{Attenello ${ }^{1}$, Opanova $M^{1}$, Wright $A^{1}$, Saruwatari $M^{2}$, Naito $K^{1}$, Chan $S^{2}$, Saito ${ }^{2}$, Nakasone $C^{3}$, Andrews $S^{3}$}

\section{Abstract}

Background: A single stage bilateral total hip arthroplasty (BTHA) utilizing the direct anterior approach (DDA) has been reported to have a similar incidence of perioperative complications as unilateral total hip arthroplasty (THA). However, previous studies have included various surgeons with differences in contraindications, protocol, technique and/or experience.

Questions/Purposes: The purpose of this retrospective review was to compare perioperative outcomes in singlestage bilateral and unilateral total hip arthroplasties via the direct anterior approach performed by a single, fellowship trained, high volume arthroplasty surgeon.

Methods: A retrospective review was completed on consecutive single-stage bilateral total hip arthroplasties performed between 2009 and 2017 and compared to consecutive unilateral total hip arthroplasties performed between 2014 and 2016. Perioperative data and complications occurring within 90 days were collected for all included patients. Student t-tests were performed to detect differences between bilateral and unilateral surgical variables.

Results: A total of 349 patients (531 hips) were included, consisting of 182 BTHA patients (364 hips) and 167 unilateral THA patients. Patients undergoing unilateral THA had significantly lower operating time, shorter length of stay, lower estimated blood loss, lower rate of transfusions and higher rate of home discharge compared to BTHA $(p<0.001)$. Complications were present in four unilateral THA patients, three requiring revision, and nine BTHA patients, three requiring revision.

Conclusions: There was no difference in complications, as well as no perioperative mortalities or systemic complications, within 90 days following surgery between unilateral and bilateral patients. Based on these results, single-stage DAA BTHA is a safe procedure to perform, and did not appear to result in higher rates of complications when compared to patients receiving a DAA unilateral THA.

\section{Background}

Total hip arthroplasty (THA) is commonly performed for the treatment of osteoarthritis, with previous research citing good clinical outcomes and survivorship, as well as consistently high patient satisfaction outcomes. These positive outcomes have increased with the introduction of the minimally invasive direct anterior approach (DAA), providing shorter recovery time, improved pain control and increased functional scores following surgery. However, previous research has reported up to $25 \%$ of patients undergoing THA for the treatment of osteoarthritis will re-

Keywords: total hip arthroplasty; complications; direct anterior approach

Level of Evidence: AAOS Therapeutic Level III 
quire a contralateral THA [1,2], with $10 \%$ of those patients having met the criteria for bilateral THA (BTHA) at initial presentation $[\underline{3}, \underline{4}]$.

A two-stage THA, in which two unilateral surgical procedures are performed, has historically been preferred due to the decreased risk of single event large blood loss, venous thromboembolic events and delays in recovery time [5]. However, current research supports the use of onestage BTHA, in which the surgical procedure is performed under one anesthetic event, reporting shorter operating times and potentially more symmetrical post-operative limb lengths [ㅁ-10]. Additionally, single-stage BTHA patients have previously been reported to have faster recovery time, with improved hip flexion and performance in activities of daily living [11].

Previous research evaluating the single-stage BTHA utilizing a DAA has reported low incidence of perioperative complications and rates of transfusions $[\underline{12}, \underline{13}]$. When comparing unilateral DAA THA and one-stage DAA BTHA, no differences were reported in perioperative complications, length of stay and discharge disposition [14]. However, sample sizes were small and data was pooled from various surgeons with differences in contraindications, protocol, technique and/or experience. The purpose of this retrospective review was to compare perioperative outcomes in single-stage DAA BTHA and unilateral DAA THA performed by a single, fellowship trained, high volume arthroplasty surgeon at a single multispecialty community institution.

\section{Materials and Methods}

This institutional review board approved study was a retrospective chart review of DAA THAs performed by a single, fellowship trained arthroplasty surgeon. Consecutive single-stage DAA BTHA performed between 2009 and 2017 were evaluated and compared to consecutive unilateral THAs performed between 2014 and 2016. For both groups, inclusion criteria for this review was all elective THAs performed for radiographic and clinical evidence of symptomatic hip osteoarthritis, rheumatoid arthritis or avascular necrosis in one or both hips. Patients with severe congenital deformity or dysplasia were not excluded. Patients undergoing THA for femoral neck fractures were excluded. A total of 349 patients (531 hips) were included, consisting of 182 BTHA patients (364 hips) and 167 unilateral THA patients. Demographic information for both groups is presented in Table 1.

All patients received a cementless total hip replacement performed through a direct anterior approach as described by Matta et al. $[15,16]$ using a specialized fracture table (Hana ${ }^{\circledR}$, Mizuho OSI, Union City, CA) and intraoperative fluoroscopy. For single-stage BTHA, the left hip was usually performed first. Patients received either general anesthesia, spinal anesthesia or a paravertebral block at the discretion of the attending anesthesiologist. All patients received an intraarticular injection mixture of Ropivicaine, Toradol and epinephrine in the amount appropriate for their weight as determined by the anesthesiologist. All

Table 1. Demographic information for all patients and by gender

\begin{tabular}{|c|c|c|c|c|c|c|c|c|c|c|c|c|c|c|c|c|}
\hline & \multicolumn{8}{|c|}{ Unilateral } & \multicolumn{8}{|c|}{ Bilateral } \\
\hline & \multicolumn{4}{|c|}{ Males } & \multicolumn{4}{|c|}{ Females } & \multicolumn{4}{|c|}{ Males } & \multicolumn{4}{|c|}{ Females } \\
\hline & \multicolumn{4}{|c|}{$\mathrm{n}=79$} & \multicolumn{4}{|c|}{$\mathrm{n}=88$} & \multicolumn{4}{|c|}{$\mathrm{n}=81$} & \multicolumn{4}{|c|}{$n=101$} \\
\hline & Mean & & SD & Range & Mean & & SD & Range & Mean & & SD & Range & Mean & & SD & Range \\
\hline Age & 65.6 & \pm & 9.6 & $(39,86)$ & 67.5 & \pm & 11.0 & $(89,23)$ & 61.7 & \pm & 10.5 & $(25,85)$ & 64.6 & \pm & 11.3 & $(25,92)$ \\
\hline $\begin{array}{l}\text { Weight } \\
(\mathrm{kg})\end{array}$ & 79.4 & \pm & 17.9 & $(37.6,142.4)$ & 71.9 & \pm & 16.9 & $(39.9,130.1)$ & 84.5 & \pm & 13.9 & $(49.0,123.8)$ & 64.7 & \pm & 11.9 & $(41.3,119.3)$ \\
\hline $\begin{array}{l}\text { Height } \\
(\mathrm{cm})\end{array}$ & 169.2 & \pm & 10.2 & $(148.6,195.6)$ & 163.3 & \pm & 9.7 & $(146.1,190.0)$ & 174.6 & \pm & 8.4 & $(147.3,190.5)$ & 159.1 & \pm & 8.4 & $(137.2,175.3)$ \\
\hline BMI & 28.2 & \pm & 5.2 & $(18.5,47.7)$ & 26.1 & \pm & 5.1 & $(16.9,39.0)$ & 27.7 & \pm & 4.0 & $(18.0,38.1)$ & 25.6 & \pm & 4.7 & $(17.6,45.1)$ \\
\hline \multicolumn{17}{|l|}{ ASA } \\
\hline 1 & \multicolumn{4}{|c|}{1} & \multicolumn{4}{|c|}{0} & \multicolumn{4}{|c|}{0} & \multicolumn{4}{|c|}{2} \\
\hline 2 & \multicolumn{4}{|c|}{38} & \multicolumn{4}{|c|}{47} & \multicolumn{4}{|c|}{42} & \multicolumn{4}{|c|}{70} \\
\hline 3 & \multicolumn{4}{|c|}{40} & \multicolumn{4}{|c|}{40} & \multicolumn{4}{|c|}{39} & \multicolumn{4}{|c|}{28} \\
\hline 4 & \multicolumn{4}{|c|}{0} & \multicolumn{4}{|c|}{1} & \multicolumn{4}{|c|}{0} & \multicolumn{4}{|c|}{1} \\
\hline
\end{tabular}

$n=$ number of patients in group; $S D=$ standard deviation; $\mathrm{kg}=$ kilograms; $\mathrm{cm}=$ centimeters

$B M I=$ body mass index $;$ ASA = American Society of Anesthesiologist classification 
patients received a multimodal preoperative pain control protocol which consisted of $200 \mathrm{mg}$ of oral Celebrex, 500 $\mathrm{mg}$ of oral Tylenol and $10 \mathrm{mg}$ of OxyContin prior to surgery. All patients received appropriate prophylactic antibiotics just prior to surgery and for 24 hours following surgery. All patients received $325 \mathrm{mg}$ of Aspirin twice daily following surgery for deep vein thrombosis chemoprophylaxis unless they could not tolerate Aspirin or if risk factors such as previous deep vein thrombosis or pulmonary embolism necessitated chemoprophylaxis with Lovenox or other anticoagulants. Following surgery, as needed oral narcotic medications and $500 \mathrm{mg}-1000 \mathrm{mg}$ of oral acetaminophen were given every six hours. Intravenous narcotic medications were used only if pain exceeded the oral pain medications given.

All patients had physical therapy initiated on the day of surgery and twice daily while hospitalized. Immediate full, unrestricted weight bearing was allowed. Patients did not have hip precautions and were specifically educated before surgery that they could perform any hip motions that they felt safe and comfortable to do at any time following surgery. Decision on time of discharge and disposition were made as a team (which included the physical therapist) and was dependent on the physical performance and ability to safely and independently perform activities of daily living and stair negotiation. Discharge to home, acute inpatient rehabilitation or skilled nursing level transfer was decided based on the functional abilities of the patient following surgery.

Patients were seen two weeks following surgery to assess wound healing, then at six weeks, three months, six months, one year and two years following surgery with plans for follow up every five years thereafter. Immediately following surgery, patients had supine anteroposterior radiographs taken in the recovery bay. Weight bearing anteroposterior pelvis radiographs as well as frog leg lateral views were taken at the six week visit. Repeat films were done at the six month evaluation and at one year and two years following surgery. Patients with unusual pain or clinically abnormal symptoms had appropriate evaluations as indicated by the specific complaints.

Surgical data reviewed included operative times (skin incision to skin closure), estimated intraoperative blood loss, postoperative or intraoperative rate of blood transfusions, length of hospital stay and discharge disposition. Perioperative complications were defined as any complication arising within 90 days following surgery. All serious postoperative complications that required repeat surgery were recorded prospectively. Thigh pain and groin pain that persisted past six weeks following surgery were also recorded prospectively and monitored. Any serious medi- cal complications such as myocardial infarctions, cerebrovascular events, deep vein thrombosis or pulmonary emboli were likewise reported. Readmissions for any reason were also recorded within 90 days following surgery.

Demographic information for both groups were organized by gender. Descriptive statistics, including means and standard deviations, for all surgical based outcomes were determined for all patients by group. Student t-tests were performed to detect differences between BTHA and unilateral THA surgical variables, with a significance level of $\mathrm{p}<0.05$.

\section{Results}

Patients undergoing unilateral THA had significantly lower operating time, shorter length of stay, lower estimated blood loss, lower rate of transfusions and higher rate of home discharge compared to BTHA $(\mathrm{p}<0.001)$ (Table 2). Complications were present in four unilateral THA patients, three requiring revision, and nine BTHA patients, three requiring revision (Table 3 ).

\section{Discussion}

Although previous studies have demonstrated the safety and efficacy of BTHA performed through the DAA [4,17], the single surgeon design of current study provides uniformity in protocol, technique and surgeon experience, thus providing a more accurate evaluation of perioperative outcomes. In the current study, complications were present in $2.1 \%$ of unilateral THA and $2.5 \%$ of BTHA, with no perioperative mortalities or systemic complications, to include cardiac or cerebrovascular events, deep vein thrombosis or pulmonary embolism.

Table 2. Comparison of surgical variables by group

\begin{tabular}{|l|r|l|l|r|l|l|l|}
\hline & \multicolumn{3}{|c|}{ Unilateral THA } & \multicolumn{3}{c|}{ Bilateral THA } & p-value \\
\hline & \multicolumn{2}{|c|}{$\mathbf{n = 1 6 7}$} & \multicolumn{2}{c|}{$\mathbf{n = 1 8 2}$} & \\
\hline & Mean & & SD & Mean & & SD & \\
\hline ORT (min) & 75.2 & \pm & 12.3 & 170.1 & \pm & 38.0 & $<0.001$ \\
\hline LOS (days) & 2.1 & \pm & 0.9 & 2.6 & \pm & 0.1 & $<0.001$ \\
\hline EBL (cc) & 239.2 & \pm & 76.6 & 402.8 & \pm & 99.4 & $<0.001$ \\
\hline Home Discharge & \multicolumn{3}{|c|}{$86.0 \%$} & \multicolumn{3}{c|}{$67.0 \%$} & $<0.001$ \\
\hline Transfusion & \multicolumn{3}{|c|}{$6.6 \%$} & \multicolumn{2}{c|}{$12.4 \%$} & $<0.001$ \\
\hline
\end{tabular}

THA = total hip arthroplasty; $S D=$ standard deviation

$n=$ number of patients; ORT = operating room time

$\min =$ minutes; $L O S=$ length of hospital stay

$E B L=$ estimated blood loss $; c c=$ cubic centimeter 
Table 3. Surgical Complications

\begin{tabular}{|l|l|l|l|l|l|l|l|l|c|}
\hline & Patient & Gender & Age & BMI & ASA & $\begin{array}{c}\text { ORT } \\
(\mathbf{m i n s})\end{array}$ & $\begin{array}{c}\text { LOS } \\
(\mathbf{d a y s})\end{array}$ & Complication & $\begin{array}{c}\text { Revision } \\
\text { Required }\end{array}$ \\
\hline Unilateral & & & & & & & & & \\
\hline & 1 & Female & 57 & 27.5 & 3 & 98 & 2 & Intraoperative Femur Fracture & No \\
\hline & 2 & Female & 73 & 20.8 & 3 & 63 & 3 & Periprosthetic Femur Fracture & Yes \\
\hline & 3 & Female & 83 & 27.3 & 2 & 73 & 7 & Periprosthetic Femur Fracture & Yes \\
\hline & 4 & Female & 54 & 17.7 & 2 & 105 & 2 & Dislocation & Yes \\
\hline & 1 & Male & 66 & 21.2 & 3 & 60 & 3 & Deep Infection & No \\
\hline & 2 & Female & 67 & 20.3 & 2 & 193 & 4 & Intraoperative Femur Fracture & No \\
\hline & 4 & Female & 77 & 24.6 & 3 & 137 & 4 & Periprosthetic Femur Fracture & Yes \\
\hline & 5 & Male & 64 & 23.4 & 2 & 128 & 2 & Superficial Infection & No \\
\hline & 6 & Male & 70 & 31.6 & 2 & 91 & 4 & Periprosthetic Femur Fracture & Yes \\
\hline & 7 & Female & 41 & 25.1 & 1 & 251 & 3 & Dislocation & No \\
\hline & 8 & Female & 77 & 25.4 & 2 & 181 & 3 & Periprosthetic Femur Fracture & Yes \\
\hline & 9 & Female & 71 & 24.8 & 3 & 164 & 2 & Superficial Infection & No \\
\hline
\end{tabular}

$B M I=$ body mass index $; A S A=$ American society of Anesthesiologist physical status classification;

$O R T=$ operating room time in minutes; $L O S=$ length of stay in days

In addition to being a large, single surgeon cohort, the inclusion criteria for this study was widely inclusive and single-stage DAA BTHA was offered to all patients with bilateral involvement regardless of age or comorbidities. This is different than previous studies, in which patients were excluded from single stage bilateral consideration based on age, preoperative anemia, pulmonary disease, renal disease and history of cardiac events $[\underline{4}, \underline{12}-14,18]$. In the current study, patients experiencing a complication did not represent the extremes in age, BMI or ASA category, challenging the concern inferred by previous studies that patients in these categories should be excluded from onestage BTHA.

All surgical outcome measures were significantly different between unilateral THA and BTHA, as expected. Operating time for BTHA patients was approximately 85 minutes per hip compared to 75 minutes for unilateral THA, with the extra time most likely due to the redrapping of the patient. Length of stay was statistically significantly longer for BTHA than unilateral THA but clinically insignificant, and more unilateral THAs were discharged directly home, highlighting the increased short term functional limitations expected in the BTHA patients. Remarkably, nearly $70 \%$ of single stage DAA BTHA patients were able to discharge directly home after an average hospital stay of only 2.6 days.

There were a number of limitations in the current study.
First, this was a retrospective evaluation of two surgial procedures. However, all procedures were performed by the same surgeon with the same standard of care protocol for each patient, limiting the influence of surgical technique and protocol on the outcomes. Additoinally, patients were not excluded from the study based on commorbidities, therefore, eliminating the patient selection as a potential bias in the results. Secondly, no long term follow up or patient outcome measures were collected in this study so no conclusion can be made about function or patient satisfaction following surgery. However, the purpose of this study was to descibe only perioperative complications occuring during the two procedures, determining if the single-stage BTHA was a safe option of patients with bilateral symptoms.

\section{Conclusion}

In this retrospective comparison, perioperative complications were present four unilateral THA patients, three requiring revision, and nine BTHA patients, three requiring revision, within 90 days following surgery. As expected, unilateral THA patients had significantly lower operating time, shorter length of stay, lower estimated blood loss, lower rate of transfusions and higher rate of home discharge compared to BTHA. Uniquely different than previ- 
ous research, exclusion criteria did not differ between the unilateral THA and BTHA groups. All patients with bilateral hip arthritis were offered single stage BTHA regardless of age or comorbidities. This is the first study to report a complication comparison in a large cohort of patients undergoing BTHA via the DAA by a single surgeon, without excluding patients of advanced age or presence of co-morbidities. Based on these results, single-stage DAA BTHA is a safe procedure to perform, and did not appear to result in higher rates of complications when compared to patients receiving a DAA unilateral THA.

\section{References}

1. Berend KR, Lombardi Jr AV, Adams JB. Simultaneous vs staged cementless bilateral total hip

2. arthroplasty: perioperative risk comparison. J Arthroplasty 2007;22(6 Suppl 2):111.

3. Lazansky MG. A method for grading hips. J Bone Joint Surg (Br) 1967;49(4):644.

4. Alfaro-Adrian J, Bayona F, Rech JA, et al. One- or two-stage bilateral total hip replacement.J

5. Arthroplast 1999;14:439.

6. Mast NH, Munoz BA, Matta J. Simultaneous bilateral supine anterior approach total hip arthroplasty: evaluation of early complications and short-term rehabilitations. Orthop Clin North Am 2009;40:351.

7. Bhan S, Pankaj A, Malhotra R. One- or two-stage bilateral total hip arthroplasty: a prospective, randomised, controlled study in an Asian population. J Bone Joint Surg (Br) 2006;88(3):298.

8. Berend ME, Ritter MA, Harty LD, Davis KE, Keating EM, Meding JB, Thong AE Simultaneous bilateral versus unilateral total hip arthroplasty: an outcomes analysis. J Arthroplasty 2005 Jun;20(4):421e6.

9. Lindberg-Larsen M, Joergensen CC, Husted H, et al. Simultaneous and staged bilateral total hip arthroplasty: a Danish nationwide study. Arch Orthop Trauma Surg 2013;133(11):1601.

10. Parvizi J, Pour AE, Peak EL, Sharkey PF, Hozack WJ, Rothman RH. One-stage bilateral total hip arthroplasty compared with unilateral total hip arthroplasty: a prospective study. J Arthroplasty 2006 Sep;21(6 Suppl. 2):26e31.

11. Parvizi J, Tarity TD, Sheikh E, Sharkey PF, Hozack WJ, Rothman RH. Bilateral total hip arthroplasty: one-stage versus two stage procedures. Clin Orthop Relat Res 2006 Dec;453:137e41.

12. Tsiridis E, Pavlou G, Charity J, Tsiridis E, Gie G, West R. The safety and efficacy of bilateral simultaneous total hip replacement: an analysis of 2063 cases. J Bone Jt Surg Br 2008 Aug;90(8):1005e12.

13. Yoshii T, Jinno T, Morita S, et al. Postoperative hip motion and functional recovery after simultaneous bilateral total hip arthroplasty for bilateral osteoarthritis. J Orthop Sci 2009;14(2):161.

14. Lanting BA, Odum SM, Cope RP, Patterson AH, Masonis JL. Incidence of perioperative events in single setting bilateral direct anterior approach total hip arthroplasty. J Arthroplasty. 2015 Mar;30(3):465-7.

15. Tamaki T, Oinuma K, Miura Y, Higashi H, Kaneyama R, Shiratsuchi H. Perioperative complication rate of one-stage bilateral total hip arthroplasty using the direct anterior approach. J Orthop Sci. 2016 Sep;21(5):658-61.

16. Parcells BW, Macknet DM, Kayiaros ST. The Direct Anterior Approach for 1-Stage Bilateral Total Hip Arthroplasty: Early Outcome Analysis of a Single-Surgeon Case Series. J Arthroplasty. 2016 Feb;31(2):434-7.

17. Matta JM, Ferguson TA. The anterior approach for hip replacement. Orthopedics 2005;28(9):927-8.

18. Matta JM, Shahrdar C, Ferguson T. Single-incision anterior approach for total hip arthroplasty on an orthopaedic table. Clin Orthop Relat Res 2005;441:115-24.

19. Weinstein MA, Keggi JM, Zatorski LE, et al. One stage bilateral total hip arthroplasty in patients > or 575 years. Orthopedics 2002;25(2):153-6.

20. Gondusky JS, Pinkos KA, Choi L, Patel JJ, Barnett S, Gorab RS. Simultaneous Bilateral Anterior Approach Total Hip Arthroplasty. Orthopedics. 2015 Jul1;38(7):e611-5.
SUBMISSION HISTORY

Submitted October 26, 2018

Reviewed November 11, 2018

Revised December 10, 2018

Accepted December 13, 2018

Published December 31, 2018

\section{AUTHOR AFFILIATIONS}

1 John Attenello, MD; Maria Opanova, MD; Anne Richardson Wright, MD; Kelvin Naito Department of Orthopedic Surgery, University of Hawai'i, 1356 Lusitana Street 6th Floor, Honolulu, $\mathrm{HI}$

2 Michele Saruwatari; Sean Chan

John A Burns School of Medicine, University of Hawai i, 651 Ilalo St, Honolulu HI 96813

3 Cass Nakasone, MD; Samantha Andrews, PhD, ATC

Bone and Joint Clinic, Straub Medical Center, 888 S King Street, Honolulu, HI 96813

(Direct inquires to Samantha Andrews, samantha.andrews@straub.net)

AUTHOR DISCLOSURES

The authors declare that there are no disclosures regarding the publication of this paper.

\section{COPYRIGHT \& OPEN ACCESS}

() 2018 Attenello, Opanova, Wright, Saruwatari, Naito, Chan, Saito, Nakasone, Andrews. All rights reserved.

Authors retain copyright and grant the journal right of first publication with the work. Reconstructive Review is an open access publication and follows the Creative Commons Attribution-NonCommercial CC BY-NC. This license allows anyone to download works, build upon the material, Foundation (JISRF). An example credit would be: "Courtesy of (senior author's name), Reconstructive Review, JISRF, Chagrin Falls, Ohio". 